\title{
Adoption of Gene Expression Profiling for Breast Cancer in US Oncology Practice for Women Younger Than 65 Years
}

\author{
Suzanne C. O’Neill, $\mathrm{PhD}^{\mathrm{a}}$; Claudine Isaacs, MDª Calvin Chao, MD' ; Huei-Ting Tsai, PhD, MPH${ }^{\mathrm{a}}$; \\ Chunfu Liu, ScDc; Bola F. Ekezue, PhDc; Nandini Selvam, PhDc; Larry G. Kessler, ScDd; \\ Marc D. Schwartz, $\mathrm{PhD}^{\mathrm{a}}$; Tania Lobo, $\mathrm{MS}^{\mathrm{a}}$; and Arnold L. Potosky, $\mathrm{PhD}^{\mathrm{a}}$
}

\begin{abstract}
Background: A number of practice guidelines incorporate the use of gene expression profiling (GEP) tests for early-stage, hormone receptor-positive, HER2-negative breast tumors. Few studies describe factors associated with GEP testing in US oncology practice. We assessed the relationship between clinical, demographic, and group-level socioeconomic variables and test use in women younger than 65 years. Patients and Methods: Data from 5 state cancer registries were linked with insurance claims data and GEP test results. We assessed rates of testing and variables associated with test use in an incident cohort of 9,444 commercially insured women younger than 65 years, newly diagnosed with stage I or II hormone receptor-positive breast cancer from 2006 through 2012. Results: Rates of testing for women with N0 disease increased from $20.4 \%$ in 2006 to $35.2 \%$ in 2011. Variables associated with higher rates of testing, beyond clinical factors such as nodal status $(P<.001)$, included being diagnosed from 2008 through 2012 versus 2006 through 2007 (adjusted odds ratio [OR], 1.67; 95\% Cl, 1.47-1.90), having preexisting comorbidities (adjusted OR, 1.35; 95\% Cl, 1.14-1.59), and higher out-of-pocket pharmacy costs (adjusted OR, $1.66 ; 95 \% \mathrm{Cl}, 1.40-1.97)$. Women younger than 50 years were more likely to be tested if they had stage I versus stage II disease $(P<.0001)$. Conclusions: In an insured population of women younger than 65 years, GEP testing increased after its inclusion in clinical practice guidelines and mounting evidence. Additional research is needed to better understand oncologists' decision not to order GEP testing for their patients who are otherwise eligible. (J Natl Compr Canc Netw 2015;13:1216-1224)
\end{abstract}

\section{Background}

A number a practice guidelines incorporate consideration of gene expression profiling (GEP) of early-stage, hormone receptor-positive, HER2negative breast tumors with existing pathologic features to refine recurrence estimates and guide treatment recommendations. ${ }^{1-4}$ More limited evidence supports its use in patients with 1 to 3 positive nodes, ${ }^{5}$ and testing is not included in clinical guidelines for these patients. The Oncotype DX Breast Cancer Assay (Genomic Health, Inc., Redwood City, CA), the most commonly used GEP test in the United States and, at the time of writing, the only GEP included in the NCCN Clinical Practice Guidelines in Oncology (NCCN Guidelines) for Breast

From ${ }^{\mathrm{a}}$ Georgetown Lombardi Comprehensive Cancer Center, Washington,

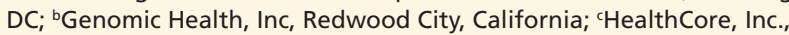
Wilmington, Delaware; and UUniversity of Washington School of Public Health, Seattle, Washington.

Submitted March 23, 2015; accepted for publication July 16, 2015. Dr. Isaacs has disclosed that she is on the speakers' bureau for Genentech Inc. Dr. Chao is an employee of and owns stock options and shares in Genomic Health, Inc. Drs. Liu, Ekezue, and Selvam are employees of HealthCore, Inc. The remaining authors have disclosed that they have no financial interests, arrangements, affiliations, or commercial interests
Cancer, ${ }^{3,6-8}$ is a 21 -gene assay that provides women and clinicians with a recurrence score (RS) result that, combined with other prognostic variables, can be used to identify women at either low, intermediate, or high risk of recurrence and estimate the benefit of adjuvant chemotherapy (to view the most recent version of the NCCN Guidelines, visit NCCN.org). ${ }^{9-16}$

Studies of GEP test adoption, and use of Oncotype DX specifically, suggest that between $20 \%$ and $50 \%$ of eligible women were tested. ${ }^{17-20}$ This rate of testing is likely aided by the incorporation of Oncotype DX testing into guidelines,,${ }^{1,2}$ and the eventual attainment of nearly universal reimbursement by health plans for guideline-informed testing. ${ }^{21,22}$ These studies have found that, although rates of testing have increased

with the manufacturers of any products discussed in this article or their competitors.

This study was funded by R01CA160671 and P30CA051008 from the National Cancer Institute. Manuscript preparation was supported by MRSG 10-110-01-CPPB from the American Cancer Society. The content is solely the responsibility of the authors and does not necessarily represent the official views of the National Cancer Institute or the National Institutes of Health.

Correspondence: Suzanne C. O'Neill, PhD, Lombardi Comprehensive Cancer Center, 3300 Whitehaven Street, NW, Suite 4100, Washington, DC 20007. E-mail: sco4@georgetown.edu 
since 2007, the year testing was first incorporated into a clinical practice guideline, the likelihood of testing varies across practice settings and is related to several clinical and demographic variables. Overall, rates of testing were highest among women with less-aggressive disease features and with estrogen and/or progesterone receptor (ER/PR)-positive, HER2-negative disease that reflects clinical practice guidelines for appropriate test use. Likewise, women with no or limited comorbidities were more likely to be tested, as were white versus African American women and those in their 50s (vs younger or older patients). ${ }^{19,20,23}$

Previous studies of GEP test use are limited by small sample sizes, ${ }^{20,23}$ lack of representativeness of community practice, ${ }^{19}$ or an inability to measure additional explanatory variables such as group-level economic variables or regional variation. Further, previous studies have focused on women of all ages. Given rates of testing are highest in women younger than 65 years, ${ }^{19,20,23,24}$ further attention is warranted to assess variables associated with testing in this population. The objectives of this study were to examine rates of GEP testing and to determine patient-level clinical, sociodemographic, and grouplevel socioeconomic variables associated with testing in an incident cohort of newly diagnosed women younger than 65 years with commercial health insurance. This study allowed us to assess test use in a large sample of newly diagnosed women receiving care across 5 states.

\section{Patients and Methods}

\section{Patient Selection and Study Cohort}

Details of data linkages are presented in supplemental eAppendix 1 (available with this article at JNCCN. org). Briefly, our linked database consists of 5 state cancer registries containing clinical and pathologic variables linked with claims data maintained by HealthCore Inc. (Wilmington, DE), an independent subsidiary to Anthem, Inc., an independent licensee of the Blue Cross and Blue Shield Association. We linked RS results through collaboration with Genomic Health, the patent holder of the Oncotype DX test. A total of 16,064 women between ages 24 and 64 years diagnosed with breast cancer (according to tumor registry data) and successfully linked with HealthCore claims were assessed for eligibility based on Anthem coverage policies for Oncotype DX testing. These coverage policies were consistent with NCCN Guidelines for Breast Cancer $^{3}$ during the study period. We selected only those women diagnosed with a first invasive breast cancer $(n=14,710)$. Based on guidelines for GEP testing, we excluded women with in situ disease, or stage III or IV or with missing stage $(n=2728)$. We also excluded women with both ER and PR missing, both ER-and PR-negative, or at least one negative/ borderline and the other missing $(n=2538)$. These exclusions resulted in a final cohort of 9,444 women diagnosed through April 30, 2012, who may have been considered for GEP testing. The participating registries, HealthCore, and Georgetown obtained all necessary IRB and HIPAA approvals for this linkage.

\section{Study Measures}

Receipt of GEP testing was identified by a linkage between HealthCore and Genomic Health test data for Anthem-covered members with breast cancer whose GEP testing was performed by Genomic Health. From registry data we obtained age at diagnosis, race/ethnicity, marital status, year of diagnosis, and diagnosis of prior primary cancers other than breast cancer, including nonmelanoma skin cancers. Staging was created using the AJCC classification for staging of breast cancer (7th edition), which is based on the TNM system. ${ }^{25}$ We obtained ER, PR, HER2, and nodal status and histologic grade from registry data. We grouped women as having either ER- and PR-positive tumors or those having either PR- or ER-positive tumors (but not both). Women with a borderline HER2 status and those with an "unknown" status were compared with those with a HER2-negative status. HER2 status was derived using the SEER Collaborative Stage Site-Specific Factor 15 (positive/negative/borderline/unknown). Furthermore, those with NO disease were compared with those with N1mic and N1 disease. Well- and moderately differentiated tumors were compared with poorly differentiated or undifferentiated tumors. From HealthCore's Integrated Research Database (HIRD), ${ }^{26}$ we ascertained 31 individual health comorbid conditions diagnosed in women from 1 year before their breast cancer diagnosis up to and including the month of diagnosis based on the Elixhauser comorbidity index. ${ }^{27}$ For each condition, we used a commonly applied algorithm that required an inpatient diagnosis and/or at least 2 outpatient 
diagnosis codes at least 30 days apart to minimize false-positives. HIRD also contains information on copays, deductibles, and coinsurance for services provided that were used to create an out-of-pocket pharmacy payment burden variable (in quintiles) over the previous 6 months before the breast cancer diagnosis. Finally, members' residential 5-digit zip codes were linked to derive sociodemographic data based on the US Census Bureau 2007-2011 American Community Survey, including median household income (in quintiles) and urban versus rural location.

\section{Statistical Analysis}

We examined the bivariate relationship between the receipt of testing and each variable. We included all variables in a multivariable logistic regression model with GEP test receipt as a binary dependent variable and all other variables as main effects. Before running our final multivariable model, we tested several hypothesized interactions individually when added to the main effects model (\{age $\times$ \{year tested, stage, comorbidity\}; \{out of pocket pharmacy costs $\} \times\{$ year tested, stage, comorbidity\}; \{year tested $\} \times\{$ stage $\}$ ). We only report those interaction terms that met our criteria for statistical significance (type I error of $0.05)$ in the final multivariable model. All tests were 2 -sided, and we used a type I error of 0.05. Because of the high number of cases with unknown HER2 status, we performed sensitivity analyses, including only those with known HER2 status $(n=4980)$ and among those with node-negative $(\mathrm{NO} ; \mathrm{n}=7054)$ versus node-positive $(\mathrm{N} 1 \mathrm{mic} / \mathrm{N} 1 ; \mathrm{n}=2390)$ disease and among eligible patients for GEP testing according to practice guidelines $(n=6546)$. We report adjusted odds ratios (ORs) and 95\% CI produced by the logistic regressions. All calculations were performed using SAS 9.3 (Cary, NC).

\section{Results}

In this cohort, 2371 women $(25.1 \%)$ received GEP testing (Table 1). The 9444 women with early-stage, hormone receptor-positive disease were evenly divided across years of diagnosis, were primarily white, were previously unaffected with cancer, had no comorbidity, and resided in urban areas. Although most tested patients had breast cancers whose clinical features aligned with clinical practice guidelines, with an overall $31.4 \%$ rate of testing across all years, $9.0 \%$ of tested women $(n=213)$ did not meet these guidelines.

Testing increased significantly over time $(P<.0001$; Figure 1$)$. Rates of testing among patients with NO disease increased from 2006 (20.4\%) to $2011(35.2 \%)$. Rates of testing among patients with N1mic disease increased substantially from 2006 $(5.1 \%)$ to $2007(18.4 \%)$, and then again in 2010 $(27.2 \%)$. Rates of testing in patients with $\mathrm{N} 1$ disease have steadily increased over time, although they remain lower $(12.3 \%)$ over all years than in patients with $\mathrm{NO}$ or N1mic disease.

Several clinical variables were independently associated with testing after adjustment for all other variables (Table 2). Characteristics associated with a lower likelihood of testing included either ER or PR positivity versus ER and PR positivity (OR, 0.69; 95\% CI, 0.59-0.81; $P<.0001)$, borderline/unknown versus HER2-negative status (OR, 0.51; 95\% CI, 0.44-0.59; $P<.0001$ ), N1mic (OR, 0.67; 95\% CI, 0.52-0.86) or N1 (OR, 0.22; 95\% CI, 0.17-0.27) compared with No disease $(P<.0001)$, and poorly or undifferentiated tumor grade versus well- or moderately differentiated grade $(\mathrm{OR}, 0.81 ; 95 \% \mathrm{CI}, 0.71-0.93 ; \mathrm{P}=.002)$. There was a significant interaction between age and stage $(P<.0001)$. The adjusted odds ratio of being tested was significantly higher for stage I versus stage II disease among women diagnosed at age 24 to 39 years (OR, 1.94; 95\% CI, 1.21-3.09) and ages 40 to 49 years (OR, 1.80; 95\% CI, 1.41-2.18), whereas no significant differences were seen in test use by stage in the older age groups (Figure 2).

The overall effect for race/ethnicity was significant $(P<.0001)$, with non-Hispanic black women the only group to be significantly less likely to be tested than non-Hispanic white women (OR, 0.61; 95\% CI, 0.45-0.82). Women with at least one comorbid condition before their cancer diagnosis were more likely to be tested than those with no comorbidities (OR, 1.35; 95\% CI, 1.141.59; $P=.0006)$. There was significant regional variation, with Georgia having the highest adjusted percentages of testing, and California (OR, 0.63; 95\% CI, 0.53-0.76) and Kentucky (OR, 0.77; 95\% $\mathrm{CI}, 0.62-0.97$ ) having the lowest testing rates. Rural patients also were less likely to be tested than urban patients (OR, 0.74; 95\% CI, 0.57-0.95; $P=.0206)$. Testing was higher among those in the top 3 quartiles of out-of-pocket pharmacy costs 
GEP in Women Younger Than 65 Years

\begin{tabular}{|c|c|c|c|c|}
\hline & & $\begin{array}{l}\text { Selected Cohort } \\
\text { Number }\end{array}$ & $\begin{array}{l}\text { Tested Cohortc } \\
\text { Row \% }\end{array}$ & $P$ Value \\
\hline Total & & 9444 & 25.1 & \\
\hline \multirow[t]{4}{*}{ Year diagnosed } & & & & $<.0001$ \\
\hline & $2006-2007$ & 3199 & 18.7 & \\
\hline & 2008-2009 & 3067 & 28.1 & \\
\hline & 2010-2012 & 3178 & 28.7 & \\
\hline \multirow[t]{5}{*}{ Age at diagnosis } & & & & $<.0001$ \\
\hline & $24-39 y$ & 660 & 17.4 & \\
\hline & $40-49$ y & 2907 & 26.3 & \\
\hline & $50-59 y$ & 3841 & 25.9 & \\
\hline & $60-64$ y & 2036 & 24.4 & \\
\hline \multirow[t]{5}{*}{ Race/Ethnicity } & & & & $<.0001$ \\
\hline & Non-Hispanic white & 7942 & 26.2 & \\
\hline & Non-Hispanic black & 400 & 17.0 & \\
\hline & Asian/Pacific Islander & 616 & 20.0 & \\
\hline & Hispanic & 386 & 20.2 & \\
\hline \multirow[t]{3}{*}{ Marital status } & & & & .52 \\
\hline & Not married & 2660 & 25.6 & \\
\hline & Married & 6614 & 24.9 & \\
\hline \multirow[t]{3}{*}{ Prior cancer } & & & & .49 \\
\hline & No & 8948 & 25.0 & \\
\hline & Yes & 496 & 26.4 & \\
\hline \multirow[t]{6}{*}{ State } & & & & .28 \\
\hline & California & 3937 & 25.1 & \\
\hline & Georgia & 1442 & 27.3 & \\
\hline & Kentucky & 745 & 25.0 & \\
\hline & New York & 1579 & 24.1 & \\
\hline & Ohio & 1741 & 24.3 & \\
\hline
\end{tabular}

Abbreviations: ER, estrogen receptor; PR, progesterone receptor.

(continued on next page)

a Missing values not included in table.

bSelected cohort: missing or unknown grade $(n=398)$, missing median household income $(n=114)$, missing area $(n=114)$, missing marital status $(n=170)$, missing race $(n=100)$.

Tested cohort: missing or unknown grade $(n=70)$, missing median household income $(n=29)$, missing area $(n=29)$, missing marital status ( $=43$ ), missing race $(n=23)$.

(OR, 1.22; 95\% CI, 1.02-1.45; OR, 1.52; 95\% CI, 1.29-1.80; and OR, 1.66; 95\% CI, 1.40-1.97, respectively) compared with those in the lowest quintile.

Results of sensitivity analyses were similar to our primary model, with a few minor exceptions, mostly resulting in a loss of statistical significance for variables due to loss of sample size in our sensitivity analyses (supplemental eAppendix 2, available with this article on JNCCN.org).

\section{Discussion}

To our knowledge, this is the largest and most representative study of US oncology practice among treated women younger than 65 years to investigate the use of Oncotype DX testing. We found that multiple clinical, demographic, and group-level economic variables are associated with the likelihood of testing in women younger than 65 years with early-stage breast cancer.

Testing rates among women with node-negative disease nearly doubled in the first few years after clinical practice guidelines incorporated GEP testing, increasing to $35 \%$ among women with NO, hormone receptor-positive, HER2-negative disease. This is a lower rate than reflected in marketing data publicly reported by Genomic Health in 2015, which suggest that testing of guideline-eligible women of all ages has continued to increase after $2011 .^{28}$ Rates of testing among women with node-positive disease also increased as the evidence base for this practice began to develop, and with the opening of the RxPONDER trial (SWOG S1007; ClinicalTrials.gov identifier: NCT01272037), which examines the effectiveness of chemohormonal versus hormonal therapy alone 
O’Neill et al

\begin{tabular}{|c|c|c|c|c|}
\hline & & $\begin{array}{l}\text { Selected Cohort } \\
\text { Number }\end{array}$ & $\begin{array}{l}\text { Tested Cohortc } \\
\text { Row \% }\end{array}$ & $P$ Value \\
\hline \multirow[t]{3}{*}{ Area } & & & & .009 \\
\hline & Rural & 479 & 20.0 & \\
\hline & Urban & 8851 & 25.4 & \\
\hline \multicolumn{2}{|c|}{ Median household income } & & & .09 \\
\hline \multicolumn{2}{|c|}{1 , lowest } & 1733 & 22.7 & \\
\hline & 2 & 1787 & 24.5 & \\
\hline & 3 & 1937 & 25.8 & \\
\hline & 4 & 1905 & 25.8 & \\
\hline & 5 , highest & 1968 & 26.4 & \\
\hline \multicolumn{2}{|c|}{ Out-of-pocket pharmacy costs } & & & $<.0001$ \\
\hline \multicolumn{2}{|c|}{$\frac{1 \text { lowest }}{1, \text { low }}$} & 1807 & 19.0 & \\
\hline & 2 & 1808 & 20.9 & \\
\hline & 3 & 1861 & 23.8 & \\
\hline & 4 & 1964 & 28.7 & \\
\hline & 5 , highest & 2004 & 32.2 & \\
\hline \multirow[t]{3}{*}{ Stage } & & & & $<.0001$ \\
\hline & $\mathrm{I}$ & 5582 & 31.6 & \\
\hline & II & 3862 & 15.7 & \\
\hline \multicolumn{2}{|c|}{ Nodal involvement } & & & $<.0001$ \\
\hline & No & 7054 & 30.2 & \\
\hline & N1mic & 609 & 19.0 & \\
\hline & N1 & 1781 & 7.1 & \\
\hline \multirow[t]{4}{*}{ HER2 status } & & & & $<.0001$ \\
\hline & Positive & 688 & 11.5 & \\
\hline & Negative & 4175 & 31.7 & \\
\hline & Borderline/ Unknown & 4581 & 21.2 & \\
\hline \multicolumn{2}{|c|}{ Hormone receptor status } & & & $<.0001$ \\
\hline \multicolumn{2}{|c|}{$\begin{array}{l}\text { ER and PR both } \\
\text { positive }\end{array}$} & 7990 & 26.4 & \\
\hline \multirow{2}{*}{\multicolumn{2}{|c|}{$\begin{array}{ll} & \text { At least } 1 \text { positive } \\
\text { Histologic grade } & \end{array}$}} & 1454 & 18.0 & \\
\hline & & & & $<.0001$ \\
\hline & $1-2$ & 6869 & 27.7 & \\
\hline & 3 & 2177 & 18.3 & \\
\hline \multicolumn{2}{|c|}{ One-year comorbidities } & & & $<.0001$ \\
\hline & 0 (ref) & 8531 & 24.1 & \\
\hline & $\geq 1$ & 913 & 34.3 & \\
\hline
\end{tabular}

Abbreviations: ER, estrogen receptor; PR, progesterone receptor.

aMissing values not included in table.

bSelected cohort: missing or unknown grade $(n=398)$, missing median household income $(n=114)$, missing area $(n=114)$, missing marital status $(n=170)$, missing race $(n=100)$.

'Tested cohort: missing or unknown grade $(n=70)$, missing median household income $(n=29)$, missing area $(n=29)$, missing marital status ( $n=43)$, missing race $(n=23)$.

for women with 1 to 3 positive nodes. ${ }^{29,30}$ Nonetheless, rates of testing remain lower for this group. Overall, time trends in test use appear to be influenced by factors such as the evidence for clinical utility, coverage by insurers, and incorporation into clinical practice guidelines. ${ }^{31}$ Future changes in adoption are likely after the results of ongoing trials and other validation studies are published, and in response to increases in patient demand for testing. Qualitative studies with oncologists suggest that they take a number of factors into consideration when ordering testing, including not only clinical variables but also patients' pretest preferences for che- motherapy $y^{32}$ and the degree of uncertainty regarding their recommendation for chemotherapy. ${ }^{33}$ Additional multimethod research is needed to further understand oncologists' decision-making processes regarding the use of GEP testing, and precision medicine more broadly, and how they involve patients in this process.

The likelihood of testing was strongly associated with clinical criteria that align with clinical practice guidelines. A minority of tested patients had disease features that made them ineligible for testing according to guidelines consistent with Anthem's policies for testing during our study period. However, some 


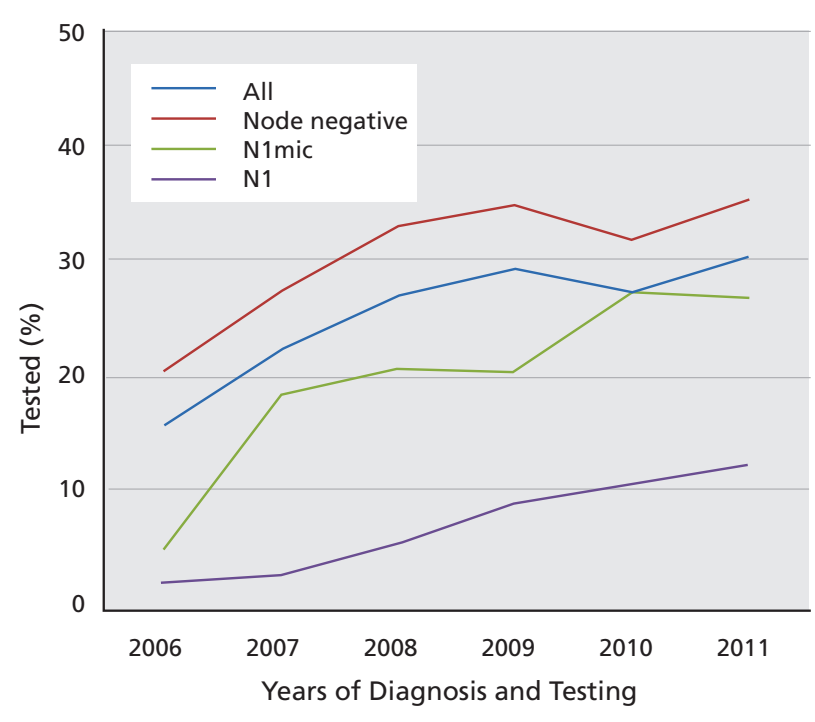

Figure 1 Rates of testing by year (2006-2011). A test of each linear trend was significant for the cohort and each subgroup $(P<.0001)$. Patients diagnosed in 2012 were not included because of incomplete data for this year.

oncologists could have determined that testing of N1 women outside of the guidelines would add value based on the current literature, ${ }^{5}$ pending the results of the RxPONDER clinical trial. ${ }^{29}$ It also is possible that these patients were discovered to have positive nodes subsequent to GEP testing. ${ }^{34}$ We also found that a small number of women with HER2-positive disease were tested outside clinical practice guidelines.

Our findings for age by stage suggest that oncologists are more likely to test younger women if they have stage I versus stage II disease. Although we found similar, nonsignificant trends for older patients, this pattern of care could reflect how oncologists use patient variables to determine the clinical utility of testing for individual patients, and therefore, whether they should order testing. For example, the presence of more aggressive stage II disease in younger women and their higher tolerance for chemotherapy-related side effects could lead oncologists to determine that chemotherapy is the optimal treatment for these patients, regardless of their RS. ${ }^{35,36}$ Additionally, in the studies that established the clinical utility of the Oncotype DX test and led to its incorporation in clinical practice guidelines, less than $10 \%$ of the women were younger than 40 years. ${ }^{13,37}$ Oncologists may therefore be generally less inclined to test patients younger than 40 years and those presenting with stage II disease, because of the potential benefit offered by chemotherapy regardless of test result.
Conversely, they could be more open to omitting

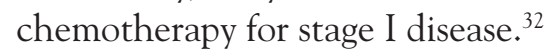

Our results also suggest that the likelihood of testing in our cohort was higher among patients with at least one preexisting comorbidity. These results differ from those of previous studies that have uniformly found that rates of testing are inversely related to the presence of comorbidites. ${ }^{19,24}$ This difference may be due to our younger cohort, because greater comorbidity may be associated with less benefit from chemotherapy, and increases the probability of adverse toxicity events from chemotherapy. ${ }^{38}$ Although GEP testing and chemotherapy may be less likely to be considered for women older than 65 years, in our cohort, the positive association of testing with comorbidity may reflect the use of the test to help identify the subgroup of women at a higher risk of chemotherapy-induced toxicity and reductions in quality of life who might safely forgo chemotherapy. ${ }^{39}$

Although our sample was large, we were limited in the inclusion of African American, Asian American/Pacific Islander, and Hispanic participants. Despite this small subsample, our results replicated several studies that found lower rates of testing among non-Hispanic black women. ${ }^{19,20,23}$ The consistency of this finding suggests that this may represent an emerging but unexplained disparity in the use of genomic medicine among women with breast cancer that requires further investigation. Although we did not have information on care settings, such as whether patients were treated in an academic or community setting, all patients in our sample were covered by commercial insurance and, therefore, should have somewhat comparable access. We adjusted for outof-pocket pharmaceutical payment burden to help mitigate possible variability in access within our covered population. Further, we believe that this is the first study to document regional variation in the ordering of GEP testing, and lower rates of testing among rural versus urban patients. Although these effects were diminished in sensitivity analyses among women with confirmed HER2 status, regional variations in other unrelated aspects of cancer care and outcomes are well documented. ${ }^{40-42}$ Regional variation in GEP testing could reflect continued clinical uncertainty or professional disagreements among oncologists regarding the utility of GEP testing.

Finally, we found that testing rates were higher for patients with greater out-of-pocket burden 
O’Neill et al

\begin{tabular}{|c|c|c|c|c|}
\hline & & OR & $(95 \% \mathrm{Cl})$ & $P$ Value \\
\hline \multirow[t]{4}{*}{ Year diagnosed } & & & & $<.0001$ \\
\hline & 2006-2007 (ref) & & & \\
\hline & $2008-2009$ & 1.67 & $(1.47-1.90)$ & \\
\hline & 2010-2012 & 1.54 & $(1.34-1.76)$ & \\
\hline \multirow[t]{5}{*}{ Race/Ethnicity } & & & & .001 \\
\hline & Non-Hispanic white (ref) & & & \\
\hline & Non-Hispanic black & 0.61 & $(0.45-0.82)$ & \\
\hline & Asian and Pacific Islanders & 0.83 & $(0.66-1.04)$ & \\
\hline & Hispanic & 0.76 & $(0.5-1.01)$ & \\
\hline \multirow[t]{3}{*}{ Marital status } & & & & .54 \\
\hline & Not married & 1.04 & $(0.9-1.16)$ & \\
\hline & Married (ref) & & & \\
\hline \multirow[t]{3}{*}{ Prior cancer } & & & & .42 \\
\hline & No (ref) & & & \\
\hline & Yes & 0.91 & $(0.7-1.14)$ & \\
\hline \multirow[t]{6}{*}{ State } & & & & $<.0001$ \\
\hline & California & 0.63 & $(0.53-0.76)$ & \\
\hline & Georgia (ref) & & & \\
\hline & Kentucky & 0.77 & $(0.62-0.97)$ & \\
\hline & New York & 0.83 & $(0.69-1.01$ & \\
\hline & Ohio & 0.91 & $(0.76-1.09)$ & \\
\hline \multirow[t]{3}{*}{ Area } & & & & .02 \\
\hline & Rural & 0.74 & $(0.57-0.95)$ & \\
\hline & Urban (ref) & & & \\
\hline \multicolumn{4}{|c|}{ Median household income (area, quintiles, low to high) } & .60 \\
\hline \multicolumn{5}{|c|}{$\begin{array}{l}\text { Median household income (area, quintiles, low to high) } \\
\qquad 1 \text {, lowest (ref) }\end{array}$} \\
\hline & 2 & 1.08 & $(0.91-1.28)$ & \\
\hline & 3 & 1.13 & $(0.95-1.34)$ & \\
\hline & 4 & 1.14 & $(0.95-1.37)$ & \\
\hline & 5, highest & 1.14 & $(0.95-1.37)$ & \\
\hline & $<.0001$ \\
\hline \multicolumn{4}{|c|}{$\begin{array}{l}\text { Out-ot-pocket pharmacy costs (quintiles, low to nigh) } \\
1 \text {, lowest (ref) }\end{array}$} & \\
\hline & 2 & 1.06 & $(0.89-1.27)$ & \\
\hline & 3 & 1.22 & $(1.02-1.45)$ & \\
\hline & 4 & 1.52 & $(1.29-1.80)$ & \\
\hline & 5, highest & 1.66 & $(1.40-1.97)$ & \\
\hline
\end{tabular}

Abbreviations: ER, estrogen receptor; GEP, gene expression profiling; OR, odds ratio; PR, progesterone receptor.

for pharmacy costs. Although we are uncertain about the specific reasons for these differences, we speculate that this finding may be partly the result of oncologists' efforts to protect patients with a higher cost-sharing burden from the cost of chemotherapy if it will not significantly lower their risk for recurrence. Overall, our results suggest that oncologists consider not only disease characteristics but also additional nonclinical factors that appear to affect who receives GEP. There is room for an additional increase of testing among patients who are otherwise eligible per clinical practice guidelines.

Our study is limited by the high proportion of patients with unknown HER2 status. We were unable to account for certain variables possibly related to test use, such as academic centers versus community centers, the specialty of the oncologist ordering the GEP test, and unmeasured patient-level variables. Finally, our study may have limited generalizability to all community practice. Our cohort includes women younger than 65 years with commercial health insurance in 5 US states. Our results reflect regional variation that might not reflect use in the US overall.

\section{Conclusions}

To our knowledge, this is the largest populationbased study to assess use of GEP testing in US oncology practice. We found that the use of testing increased substantially after inclusion in clinical practice guidelines. Our results suggest that there are many eligible patients who are not tested. Although it appears that oncologists have incorporated testing for selected patients, rates of 
GEP in Women Younger Than 65 Years

\section{Table 2 Multivariable Model of Variables Associated With GEP Testing (cont.)}

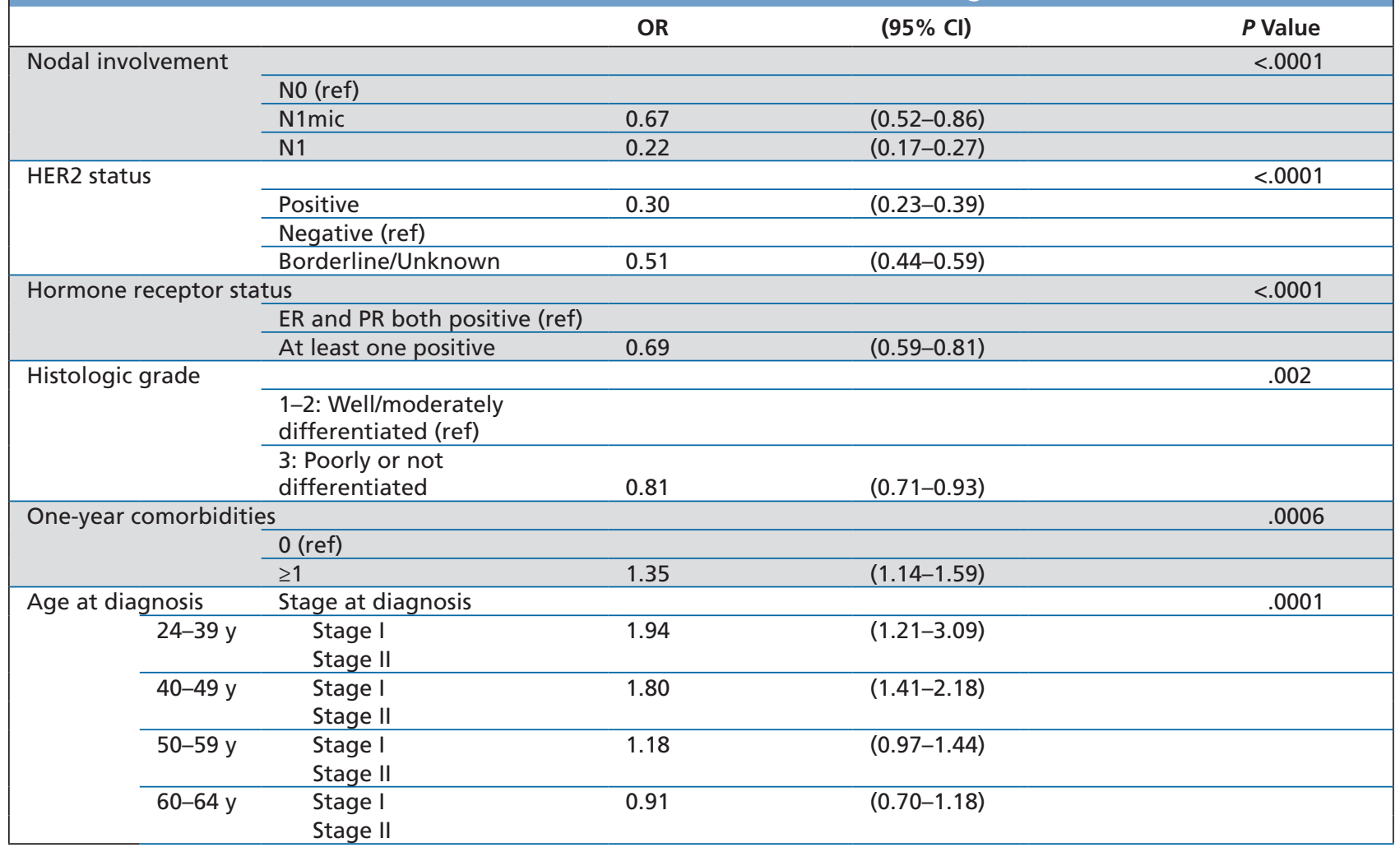

Abbreviations: ER, estrogen receptor; GEP, gene expression profiling; OR, odds ratio; PR, progesterone receptor.

testing are associated with variables associated with the clinical practice guidelines for testing (hormonal status, nodal involvement) and the clinical utility of testing based on the potential that test results will inform treatment decision-making (age, stage, comorbidities). Additional variables associated with rates of testing, including race and out-of-pocket pharmacy costs, and regional variation suggest that

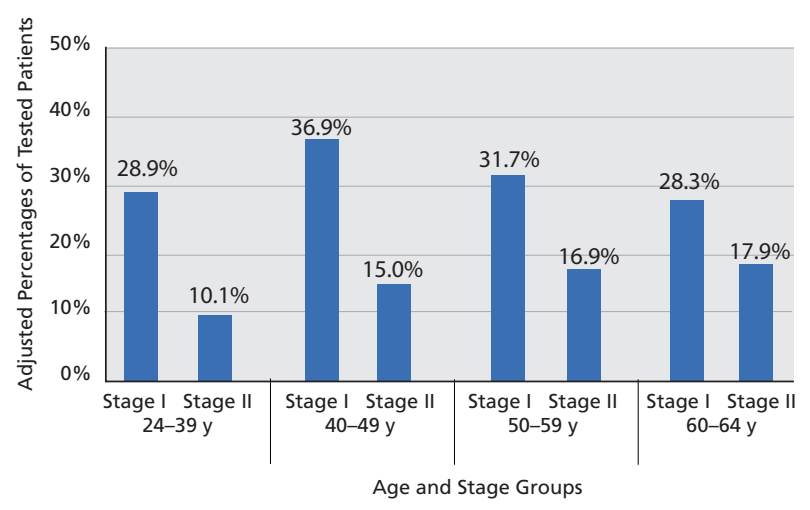

Figure 2 Interaction between age and stage among tested women. Adjusted odds of being tested was significantly higher for stage I versus stage II disease among women diagnosed at younger than 50 years. There were no significant differences in test use by stage in women aged 50 years and older. testing might not be evenly disseminated to eligible patients. Ongoing trials will help to further establish the clinical utility of testing in these subgroups and likely stimulate greater dissemination of the test in practice.

\section{Acknowledgments}

The authors would like to thank the following persons for facilitating the linkage of cancer registry data for this project: Dr. Tom Tucker and Ms. Jaclyn Nee, Kentucky Cancer Registry; Ms. Lynn Giljahn, Ohio Department of Health; Dr. Maria J. Schymura and Ms. Amy Kahn, NY State Tumor Registry; Dr. A. Rana Bayakly, Georgia Comprehensive Cancer Registry, and Dr. Kevin Ward, Georgia Center for Cancer Statistics; and Drs. Rosemary Cress and Arti Parikh-Patel, California Cancer Registry.

\section{References}

1. Goldhirsch A, Winer EP, Coates AS, et al. Personalizing the treatment of women with early breast cancer: highlights of the St Gallen International Expert Consensus on the Primary Therapy of Early Breast Cancer 2013. Ann Oncol 2013;24:2206-2223. 
2. Harris L, Fritsche H, Mennel R, et al. American Society of Clinical Oncology 2007 update of recommendations for the use of tumor markers in breast cancer. J Clin Oncol 2007;25:5287-5312.

3. Gradishar WJ, Anderson BO, Balassanian R, et al. NCCN Clinical Practice Guidelines in Oncology: Breast Cancer. Version 3.2015. Available at: NCCN.org. Accessed January 5, 2015.

4. Senkus E, Kyriakides S, Penault-Llorca F, et al. Primary breast cancer: ESMO Clinical Practice Guidelines for diagnosis, treatment and follow-up. Ann Oncol 2013;24(Suppl 6):vi7-23.

5. Dowsett M, Cuzick J, Wale C, et al. Prediction of risk of distant recurrence using the 21-gene recurrence score in node-negative and node-positive postmenopausal patients with breast cancer treated with anastrozole or tamoxifen: a TransATAC study. J Clin Oncol 2010;28:1829-1834.

6. Carlson JJ, Roth JA. The impact of the Oncotype Dx breast cancer assay in clinical practice: a systematic review and meta-analysis. Breast Cancer Res Treat 2013;141:13-22.

7. Evaluation of Genomic Applications in Practice and Prevention (EGAPP) Working Group. Recommendations from the EGAPP Working Group: can tumor gene expression profiling improve outcomes in patients with breast cancer? Genet Med 2009;11:66-73.

8. Genomic Health announces third quarter 2014 financial results and reports continued growth in U.S. invasive breast cancer business [press release]. Redwood City, CA: Genomic Health Inc.; November 4, 2014. Available at: http://investor.genomichealth.com/releaseDetail.cfm?releaseID=880398. Accessed January 5, 2015.

9. Mamounas EP, Tang G, Fisher B, et al. Association between the 21-gene recurrence score assay and risk of locoregional recurrence in node-negative, estrogen receptor-positive breast cancer: results from NSABP B-14 and NSABP B-20. J Clin Oncol 2010;28:1677-1683.

10. Goldstein LJ, Gray R, Badve S, et al. Prognostic utility of the 21-gene assay in hormone receptor-positive operable breast cancer compared with classical clinicopathologic features. J Clin Oncol 2008;26:4063-4071.

11. Hornberger J, Alvarado MD, Rebecca C, et al. Clinical validity/utility, change in practice patterns, and economic implications of risk stratifiers to predict outcomes for early-stage breast cancer: a systematic review. J Natl Cancer Inst 2012;104:1068-1079.

12. Lyman GH, Cosler LE, Kuderer NM, Hornberger J. Impact of a 21-gene RTPCR assay on treatment decisions in early-stage breast cancer: an economic analysis based on prognostic and predictive validation studies. Cancer 2007;109:1011-1018.

13. Paik $S$, Shak $S$, Tang $G$, et al. A multigene assay to predict recurrence of tamoxifen-treated, node-negative breast cancer. $\mathrm{N}$ Engl J Med 2004;351:2817-2826

14. Sparano JA, Paik S. Development of the 21 -gene assay and its application in clinical practice and clinical trials. J Clin Oncol 2008;26:721-728.

15. Tang G, Shak S, Paik S, et al. Comparison of the prognostic and predictive utilities of the 21-gene Recurrence Score assay and Adjuvant! for women with node-negative, ER-positive breast cancer: results from NSABP B-14 and NSABP B-20. Breast Cancer Res Treat 2011;127:133-142.

16. Tang G, Cuzick J, Costantino JP, et al. Risk of recurrence and chemotherapy benefit for patients with node-negative, estrogen receptor-positive breast cancer: recurrence score alone and integrated with pathologic and clinical factors. J Clin Oncol 2011;29:4365-4372.

17. DeFrank JT, Salz T, Reeder-Hayes K, Brewer NT. Who gets genomic testing for breast cancer recurrence risk? Public Health Genomics 2013;16:215-222.

18. Genomic Health Inc. Oncotype DX Frequently Asked Questions (FAQs). Available at: http://www.mybreastcancertreatment.org/en-US/ LearnAboutOncotypeDX/FAQPage.aspx\#.VIisvrctD9J. Accessed January $5,2015$.

19. Hassett MJ, Silver SM, Hughes ME, et al. Adoption of gene expression profile testing and association with use of chemotherapy among women with breast cancer. J Clin Oncol 2012;30:2218-2226.

20. Lund MJ, Mosunjac M, Davis KM, et al. 21-gene recurrence scores: racial differences in testing, scores, treatment, and outcome. Cancer 2012;118:788-796.

21. Anthem. Gene expression profiling for managing breast cancer treatment [medical policy]. Available at: http://www.anthem.com/medicalpolicies/ policies/mp_pw_a049879.htm. Accessed January 5, 2015.
22. Genomic Health Inc. Paying for the Oncotype DX test. Available at: http://www.mybreastcancertreatment.org/en-US/LearnAboutOncotypeDX/ InsuranceAndOncotypeDX.aspx\#.VKsQdLctD9J. Accessed January 5, 2015.

23. Guth AA, Fineberg S, Fei K, et al. Utilization of Oncotype DX in an inner city population: race or place? Int J Breast Cancer 2013;2013:653805.

24. Chen C, Dhanda R, Tseng WY, et al. Evaluating use characteristics for the oncotype $\mathrm{dx} 21$-gene recurrence score and concordance with chemotherapy use in early-stage breast cancer. J Oncol Pract 2013;9:182-187.

25. Edge S, Byrd DR, Compton CC, et al, eds. AJCC Cancer Staging Manual. 7th ed. New York, NY: Springer; 2010.

26. HealthCore. HealthCore Research Integrated Database. Available at: http:// www.healthcore.com/research_environment. Accessed January 5, 2015.

27. Elixhauser A, Steiner C, Harris DR, Coffey RM. Comorbidity measures for use with administrative data. Med Care 1998;36:8-27.

28. Genomic Health to present at the 33rd Annual J.P. Morgan Healthcare Conference [press release]. Redwood City, CA. Genomic Health Inc.; January 5, 2015. Available at: http://investor.genomichealth.com/ releaseDetail.cfm?releaseID=889415. Accessed January 5, 2015.

29. Ramsey SD, Barlow WE, Gonzalez-Angulo AM, et al. Integrating comparative effectiveness design elements and endpoints into a phase III, randomized clinical trial (SWOG S1007) evaluating oncotypeDXguided management for women with breast cancer involving lymph nodes. Contemp Clin Trials 2013;34:1-9.

30. Wong WB, Ramsey SD, Barlow WE, et al. The value of comparative effectiveness research: projected return on investment of the RxPONDER trial (SWOG S1007). Contemp Clin Trials 2012;33:1117-1123.

31. Dubois RW, Lauer M, Perfetto E. When is evidence sufficient for decisionmaking? A framework for understanding the pace of evidence adoption. J Comp Eff Res 2013;2:383-391.

32. Spellman E, Sulayman N, Eggly S, et al. Conveying genomic recurrence risk estimates to patients with early-stage breast cancer: oncologist perspectives. Psychooncology 2013;22:2110-2116.

33. Bombard Y, Rozmovits L, Trudeau M, et al. The value of personalizing medicine: medical oncologists' views on gene expression profiling in breast cancer treatment. Oncologist 2015;20:351-356.

34. Stemmer SM, Klang SH, Ben-Baruch N, et al. The impact of the 21-gene Recurrence Score assay on clinical decision-making in node-positive (up to 3 positive nodes) estrogen receptor-positive breast cancer patients. Breast Cancer Res Treat 2013;140:83-92.

35. Kimmick G. Adjuvant chemotherapy for breast cancer in older women: emerging evidence to aid in decision making. Curr Treat Options Oncol 2011;12:286-301.

36. Spano JP, Falandry C, Chaibi P, Freyer G. Current targeted therapies in breast cancer: clinical applications in the elderly woman. Oncologist 2011;16:1144-1153

37. Habel LA, Shak S, Jacobs MK, et al. A population-based study of tumor gene expression and risk of breast cancer death among lymph node-negative patients. Breast Cancer Res 2006;8:R25.

38. Chia VM, Page JH, Rodriguez R, et al. Chronic comorbid conditions associated with risk of febrile neutropenia in breast cancer patients treated with chemotherapy. Breast Cancer Res Treat 2013;138:621-631.

39. Hall PS, McCabe C, Stein RC, Cameron D. Economic evaluation of genomic test-directed chemotherapy for early-stage lymph node-positive breast cancer. J Natl Cancer Inst 2012;104:56-66.

40. Hines R, Markossian T, Johnson A, et al. Geographic residency status and census tract socioeconomic status as determinants of colorectal cancer outcomes. Am J Public Health 2014;104:e63-71.

41. Monson JR, Probst CP, Wexner SD, et al. Failure of evidence-based cancer care in the United States: the association between rectal cancer treatment, cancer center volume, and geography. Ann Surg 2014;260:625-631.

42. Watanabe-Galloway S, Zhang W, Watkins $K$, et al. Quality of end-of-life care among rural Medicare beneficiaries with colorectal cancer. J Rural Health 2014;30:397-405. 